\title{
Multiculturalisme, interculturalisme, antiracisme : le traitement de l'altérité
}

Multiculturalism, Interculturalism, Antiracism: Analysing Otherness

Multiculturalismo, interculturalidad, antirracismo : el tratamiento de la

alteridad

Micheline Labelle

\section{(2) OpenEdition}

Journals

\section{Édition électronique}

URL : https://journals.openedition.org/remi/7255

DOI : $10.4000 /$ remi.7255

ISSN : $1777-5418$

Éditeur

Université de Poitiers

Édition imprimée

Date de publication : 1 juin 2015

Pagination : 31-54

ISBN : 979-10-90426-25-2

ISSN : 0765-0752

Référence électronique

Micheline Labelle, « Multiculturalisme, interculturalisme, antiracisme : le traitement de l'altérité », Revue européenne des migrations internationales [En ligne], vol. 31 - n² | 2015, mis en ligne le 01 juin

2018, consulté le 14 avril 2022. URL : http://journals.openedition.org/remi/7255 ; DOI : https://doi.org/ 10.4000/remi.7255 


\section{Multiculturalisme, interculturalisme, antiracisme : le traitement de l'altérité}

\section{Micheline Labelle ${ }^{1}$}

Au sein des pays occidentaux, les modèles d'intégration varient en raison de particularités nationales. II en va de même des représentations de la nation et de la terminologie avec laquelle on identifie et catégorise les majorités et les minorités. Par ailleurs, force est de constater un décalage croissant entre les thèses qui annoncent la fin de l'État-nation dans un monde globalisé et le discours des États qui cherchent à intégrer leurs immigrants et leurs minorités, ainsi qu'à revaloriser leur citoyenneté et leur identité nationale. Le concept de modèle national présente des difficultés particulières dans les États multinationaux (Martiniello et Adam, 2013) dont les philosophies politiques diffèrent de celles des pays dits assimilationnistes (Martiniello et Simon, 2005 : 15). Ainsi, dans le cas canadien, des variantes, voire des divergences, différencient ces modèles. L'interprétation, la désignation et la mesure de l'ethnicité/diversité posent des défis particuliers dans ce contexte précis. Comme l'écrit Monica Boyd : " La terminologie utilisée est généralement tributaire du rôle des idéologies à la base de chaque État-nation sur le projet d'édification nationale propre à chaque pays, de même que l'intégration de l'ethnicité à ce projet d'édification nationale " (Boyd, $1993: 598)$.

Cet article porte sur les lignes de tension entre le multiculturalisme, I'interculturalisme et l'antiracisme et examine leur traitement respectif du rapport majorité/minorités ethnoculturelles et racisées. II ne traite en aucun cas du rapport avec les peuples autochtones dont la situation minoritaire exige un cadre d'analyse absolument différent. Le multiculturalisme et l'interculturalisme renvoient à une philosophie politique de reconnaissance de la diversité, à un ensemble de dispositifs juridiques, politiques et institutionnels d'aménagement de cette diversité ou à la stricte pluralité démographique, trois niveaux d'analyse à ne pas confondre (Labelle, 2008). Quant à l'antiracisme, il désigne l'ensemble des actions citoyennes et des politiques publiques visant l'élimination du racisme tant au plan des interactions personnelles que des structures sociales et des institutions (Labelle, $2011: 45$ ).

Dans une première partie, je montrerai comment les modèles du multiculturalisme et de l'interculturalisme font débat entre eux ou sont jugés carrément 
obsolètes et inopérants par les adeptes des théories "post " de tous genres qui se déploient aux marges du champ traditionnel de l'immigration et des "études ethniques ". Les deux parties suivantes concernent les politiques publiques du multiculturalisme canadien et de l'interculturalisme québécois, lequel tente de se consolider comme modèle attractif dans un contexte de nation minoritaire. En conclusion, je ferai ressortir les enjeux analytiques et politiques rattachés à ces questions.

\section{Multiculturalisme, interculturalisme et antiracisme : mise en regard}

\section{Multiculturalisme et interculturalisme}

Depuis les années 1990, le multiculturalisme a fait l'objet d'évaluations discordantes. Pour Kymlicka la philosophie qui sous-tend les politiques publiques du multiculturalisme cherche à renforcer les pratiques culturelles des groupes minoritaires dans l'espoir que cela conforte leur participation au sein de la société (Kymlicka, 2010). Pour d'autres, le multiculturalisme d'État aurait minimisé le racisme et isolé les conflits ethniques et culturels des autres formes de conflits (entre les sexes, entre le capital et le travail, entre le premier monde et le tiers-monde, etc.), tout en servant à détourner l'attention de la restructuration de l'État providence et de l'exploitation des marchés du cheap labour à l'échelle mondiale (Bennett, 1998 : 6 ; Salée, 2007 ; Thobani, 2007). D'autres encore déplorent l'auto-marginalisation des minorités racisées qui se réfugient dans le communautarisme, l'essentialisation et la survalorisation de l'ethnicité et des cultures d'origine et plaident pour l'assimilation. Ils se dressent contre les demandes d'accommodements fondées sur des motifs politico-religieux dont ils observent la multiplication au cours de la dernière décennie, provoquant d'indéniables conflits de valeurs et de droits. Au final, on assisterait à un repli du multiculturalisme (Alexander, 2013 ; Brubaker, 2001 ; Joppke, 2001 ; Labelle, 2008 ; Wieviorka, 1998 et 2011).

D'où un certain rappel à l'ordre émanant des théoriciens du multiculturalisme critique. Bikhu Parekh, par exemple, recommande un dialogue bifocal entre majorités et minorités. Si toutes les cultures sont dignes de respect, la majorité de la société d'accueil a cependant le droit de défendre ses "valeurs constitutives " (core common values) face à une minorité dont les pratiques lui semblent inacceptables (Parekh, $2006: 271$ ). Les sociétés ayant toutes des structures sociales, une histoire, des traditions et des valeurs qui leur sont propres, ce socle d'acquis doit constituer le point de départ de tout dialogue (Ibid. : 267). Pour Francesco Fistetti, le multiculturalisme renvoie à la coexistence des cultures au sein d'un même espace géopolitique. L'interculturalité, combat l'ethnocentrisme. Elle est sensible aux conflits entre cultures et au sein des cultures, de même qu'à leur résolution pacifique par le biais du "dialogue culturel complexe " (Fistetti, 2008 : 132-133), notion empruntée à Seyla Benhabib qui distingue les théoriciens de la démocratie des théoriciens du multiculturalisme selon les conceptions qu'ils véhiculent de la culture. Les premiers supportent les luttes pour la reconnaissance et les mouvements identitaires dans la mesure où ils visent l'inclusion démocratique, la justice sociale et politique et la fluidité culturelle (Benhabib, 2002 : IX). 
La polémique soulevée, en 2008, par le Livre blanc sur le dialogue interculturel du Conseil de l'Europe mérite que I'on s'y arrête. Le Conseil s'engageait nettement en faveur de l'interculturalisme. Tout en affirmant que " la gestion démocratique d'une diversité culturelle grandissante en Europe - ancrée dans l'histoire de notre continent et amplifiée par la mondialisation - est devenue, depuis quelques années, une priorité ", le Conseil se demandait si les individus seront un jour appelé à vivre dans des " communautés séparées, caractérisées au mieux par la coexistence de majorités et de minorités aux droits et responsabilités différenciés, vaguement reliées entre elles par l'ignorance mutuelle et les stéréotypes ? " (/bid. : 4). Le dialogue interculturel est défini comme " un processus d'échanges de vues, ouvert, respectueux et basé sur la compréhension mutuelle, entre des individus et des groupes de différentes origines et traditions ethniques, culturelles, religieuses et linguistiques, dans un esprit de compréhension et de respect mutuels " (Ibid. : 19). L'approche interculturelle éviterait les positions extrêmes de l'assimilation et du communautarisme : "Contrairement à l'assimilation, elle reconnaît que les pouvoirs publics doivent être impartiaux - au lieu de prendre pour seule norme le système de valeurs de la majorité - afin d'éviter les tensions entre communautés. Toutefois, contrairement au communautarisme, elle prône des normes communes et exclut le relativisme moral " (Ibid. : 20).

Mais l'interculturalisme n'a pas que des partisans et il fait aussi débat. En 2012, le Journal of Intercultural Studies y consacre un dossier. Selon Meer et Modood, les caractéristiques positives de l'interculturalisme (la communication, la reconnaissance d'identités dynamiques, la promotion de l'unité) se retrouvent également dans le multiculturalisme. Et pourtant, le multiculturalisme " presently surpasses interculturalisme as a political orientation that is able to recognise that social life consists of individuals and groups, and that both need to be provided for in the formal and informal distribution of powers, as well as reflected in an ethical conception of citizenship, and not just an instrumental one» (Meer et Modood, 2012 : 92). Le multiculturalisme répondrait surtout aux revendications des groupes issus de l'immigration que l'État et le " groupe dominant " ont le devoir d'accommoder. II s'agit ici de "cultures minoritaires" définies en termes de "race", d'ethnicité ou de religion (à noter ici la confusion catégorielle). II ne concerne pas les nations autochtones dont les revendications politiques ne peuvent être réduites à celles des immigrants. En dépit des efforts de Will Kymlicka pour conceptualiser le multiculturalisme comme un multinationalisme, la signification dominante du multiculturalisme concerne essentiellement les post-immigration groups (/bid. : 181).

Deux commentateurs répliquent de façon acerbe. Kymlicka, dont les travaux ont une grande influence sur la politique du multiculturalisme canadien, qualifie de faibles et de futiles le Livre Blanc du Conseil de l'Europe et le rapport de I'UNESCO (Investir dans la diversité culturelle et le dialogue interculturel, 2009). II se demande si les auteurs et les "policy makers " consultés sont conscients des conséquences de leur virage à l'interculturalisme. En sacrifiant le multiculturalisme pour renforcer l'engagement envers la diversité, cette stratégie présente des risques. Jouer la carte antimulticulturaliste risque de provoquer des opinions anti-diversité. Qui plus est, l'interculturalisme est une notion si vague qu'on peut même défendre l'assimilation en son nom (Kymlicka, 2012 : 233-334). Quant à Michel Wieviorka, il souligne l'ethnocentrisme de Meer 
et Modood qui omettent de référer aux travaux des chercheurs francophones du Sud et passent sous silence les problèmes soulevés par le multiculturalisme. D'abord les différences culturelles sont autant affaire d'invention et de transformation que de tradition. Négliger ce fait risque d'encourager une vision essentialiste et immuable des cultures. S'il y a lieu d'accorder des droits particuliers, ce doit être à des personnes et non à des minorités, et le multiculturalisme n'est admissible que dans la mesure où les membres de ces minorités acceptent pleinement la démocratie et respectent les droits de la personne. Ensuite, le multiculturalisme ne peut accommoder toutes les différences. À titre d'exemple, le multiculturalisme canadien ne peut répondre aux revendications des Québécois qui réclament l'indépendance, ni aux Premières Nations qui militent en faveur d'une plus grande autonomie gouvernementale. II ne répond pas non plus aux attentes d'immigrants qui ne veulent pas être considérés du point de vue de leur différence culturelle. Troisièmement, le multiculturalisme a été fondé sur un nationalisme méthodologique qui est périmé à l'époque de la mondialisation. Enfin, le multiculturalisme traite de cultures et non pas de religions. La question du rôle que doit jouer la religion dans un pays démocratique relève non pas du multiculturalisme, mais d'une politique de la laïcité ou de secularism. II ne peut pas non plus s'appliquer à la situation des groupes racisés, le principal problème n'étant pas ici celui de la reconnaissance identitaire, mais celui du racisme et de la discrimination. Dans ces cas, le multiculturalisme n'est pas pertinent (Wieviorka, 2012 : 227 ; Calhoun et Wieviorka : 2013). Pourtant Wieviorka persiste à défendre un multiculturalisme bien tempéré (2011), inspiré des tentatives de conciliation avec le républicanisme.

\section{Le post-multiculturalisme}

Parallèlement à ce type de débats opposant deux modèles d'intégration, les postcolonial studies, les transnational studies, les citizenship studies, les whiteness studies, les mobility studies, les borders studies remettent en cause le champ même des études ethniques et du multiculturalisme. Cette évolution des paradigmes prend sa source dans les cultural studies qui ont contribué à subvertir les définitions essentialistes de l'ethnicité, lesquelles sont au fondement du discours sur le multiculturalisme. Les courants subséquents et contemporains insistent sur le caractère situationnel, construit et fluide des " options ethniques ", leur porosité et leur créolisation. Pour éviter l'écueil de visions convenues, la tendance actuelle est d'ailleurs de substituer à l'ethnicité les termes de diversité et de super-diversité, dans les champs du savoir et des politiques publiques.

De leur côté, les théoriciens du transnationalisme et de la mobilité se sont appliqués à dénoncer le nationalisme méthodologique stato et euro-centré qui a longtemps imprégné la recherche, alors que l'État-nation ne représente plus, selon plusieurs d'entre eux, un contexte central et adéquat pour l'étude empirique de la migration internationale et des modèles d'intégration (Faist, 2012 ; Amelina et Faist, 2012 ; Boccagni, 2012 ; Glick, 2010 ; Glick Schiller et Salazar, 2013).

La citoyenneté offre également matière à réinterprétation, qu'il s'agisse de la façon de concevoir les droits et devoirs, I'identité, I'allégeance et l'appartenance à l'État-nation (Neveu et al., 2012 ; Isin, 2012 ; Schahar, 2014). Ainsi, la " colonia- 
lité du pouvoir "(concept emprunté à Anibal Quijano), renvoie selon Ramon Grosfoguel à un système tissé de formes multiples et hétérogènes de hiérarchies/ dispositifs sexuels, politiques, épistémiques, économiques, spirituels, linguistiques et raciaux de domination et d'exploitation à l'échelle mondiale (2005:57). La réinvention de la citoyenneté passe donc par la reconnaissance des effets des " formes de domination et d'exploitation après la disparition des administrations coloniales produites par les structures et les cultures du système-monde capitaliste/patriarcal moderne/colonial " et dont l'influence persiste dans divers domaines. Les projets citoyens de libération doivent s'inspirer d'un " universel radical décolonial anti-systémique diversel "( $/$ bid. : 70). On notera que Grosfoguel pense la hiérarchisation en termes de binômes : hommes/femmes, citoyens/non-citoyens, Blancs/Noirs, etc. Cette façon de hiérarchiser me semble en contradiction avec la signification du terme "diversel " créé par l'écrivain antillais Édouard Glissant pour décrire les processus d'entrecroisement des identités culturelles et linguistiques propres aux Caraïbes, et aux Amériques. En effet, la construction sociale de la " race " tout en s'inscrivant dans un univers colonial a été l'objet de multiples déclinaisons selon la classe sociale et les projets politiques, par exemple celui de la négritude (Labelle, 1978, 1987).

Dans la foulée des racial studies, I'antiracisme constitue un autre terrain de contestation du multiculturalisme, de l'interculturalisme et des études ethniques. Les travaux précurseurs du colonialisme interne avaient établi une distinction fondamentale entre minorités raciales et minorités ethniques. Dans le premier cas, le statut d'entrée dans les Amériques a résulté de la coercition et de l'esclavage ; dans le second, d'un projet migratoire volontaire. Aujourd'hui, un nombre important de chercheurs nord-américains estiment que la " race " demeure profondément ancrée dans les modes de pensée et d'action et qu'elle s'avère une composante centrale de l'identité des minorités racisées et de leurs pratiques de résistance (Wallis et Fleras, 2009 : X). Selon eux, "la race compte " dans la réalité et dans le discours scientifique, et elle demeure une catégorie d'analyse légitime (American Sociological Association, 2003 ; Lentin et Titley, 2014). Pour les tenants des whiteness studies nommer la " race " est inséparable de la lutte contre le racisme, même si elle relève du mythe social. Ici, on n'hésite pas à parler d'identité blanche, de privilège blanc, de domination blanche, de suprématie blanche, de norme blanche. La whiteness (blancheur, blanchitude) implique que l'on s'interroge sur " les spécificités de l'expérience sociale liée au fait d'être blanc, par rapport à celle découlant d'une identité noire, asiatique, ou indienne par exemple "(Garner, 2007 : 58). Ce courant analytique intéresse de plus en plus les chercheurs francophones (Garner et Fassin, 2013), courant dont se dissocient ceux qui estiment (et dont je suis) que l'on peut étudier et combattre le racisme sans recourir à la notion de " race " dont elle est le produit idéologique, sans négliger pour autant la dynamique des processus de racisation de l'altérité2. 


\section{Le rapport majorité/minorités : les mots pour le dire}

Les narratifs dont je viens de faire état opposent généralement une majorité dominante à des minorités désignées comme culturelles, ethniques, raciales ou religieuses, sujettes " en soi " à l'oppression et à la discrimination ; deux blocs monolithiques indifférenciés. C'est oublier qu'une majorité démographique peut être dominée (le cas des Bantous sous l'apartheid), qu'une majorité peut se considérer ou être considérée comme une minorité nationale (le cas québécois), qu'une minorité démographique peut être dominante sur le plan économique et politique. En outre, ce discours contribue à reproduire une représentation homogène des minorités, quelles qu'elles soient, en omettant de soulever la question de leur hétérogénéité interne, d'ordre social et politique.

L'effet de mode de la super-diversité rend la catégorisation des minorités encore plus complexe. Cette notion renvoie au jeu dynamique de variables " among an increased number of new, small and scattered, multiple-origin, transnationnaly connected, socio-economically differentiated and legally stratified immigrants " (Vertovec, 2007 : 1024). Elle s'inscrit dans le courant de l'intersectionalité qui vise à souligner le caractère multidimensionnel des oppressions fondées sur des catégorisations de race, de genre, d'origine, de sexe, de classe et comment leur combinaison s'articule dans des contextes différents. De surcroît, les catégories d'analyse basées sur la religion connaissent un essor remarquable. En Amérique du Nord, on parle de plus en plus des "communautés musulmanes "(Antonius, 2012). L'entrée en scène du religieux change considérablement la donne et contribue à un nouveau brassage catégoriel. La religion a tendance à remplacer la langue " as the cutting edge of contestation over the political accommodation of cultural difference- a striking reversal of the longer-term historical process through which language had previously displaces religion as the primary focus of contention " (Brubaker, 2013b : 1).

Ce bref tour d'horizon aura permis de constater que les théoriciens d'horizons divers persistent à reproduire une représentation polarisée du rapport majorité/ minorités, bien que certains soutiennent la thèse de la fin des majorités étant donné la mixité et la très grande diversité des cités globales du monde actuel. Examinons maintenant les tensions entre les politiques publiques du multiculturalisme, de l'interculturalisme et de l'antiracisme, dans les cas canadien et québécois. Comment se différencient ces modèles d'intégration ? Comment est pensé le rapport majorité/minorités, quelle catégorisation et quel lexique sont utilisés dans ces contextes sociaux particuliers ?

\section{Du multiculturalisme et de I'antiracisme au Canada}

Historiquement, l'idéologie raciste est partie constitutive du colonialisme qui a présidé à la création et au développement du Canada. Considérée comme I'un des principes fondateurs de la société canadienne, elle a servi de pierre d'assise à la législation, au système judiciaire, aux politiques d'immigration, aux politiques sociales et au recensement. Après la Seconde Guerre mondiale, le Canada a dû modifier son discours et ses pratiques, sous l'influence de facteurs internationaux et de considérations commerciales portant sur les liens avec les anciennes colonies de l'Empire britannique ayant acquis leur indépendance. De 
plus, l'État canadien devait faire face à la montée du mouvement indépendantiste québécois et des revendications d'autonomie gouvernementale des nations autochtones.

\section{Le multiculturalisme au Canada}

Instituée par le gouvernement canadien en 1962, la Commission royale d'enquête sur le bilinguisme et le biculturalisme fut chargée " de recommander les mesures à prendre pour que la Confédération canadienne se développe d'après le principe de l'égalité entre les deux peuples qui l'ont fondée, compte tenu de l'apport des autres groupes ethniques à l'enrichissement culturel du Canada, ainsi que des mesures à prendre pour sauvegarder cet apport ". En 1969, la Déclaration sur le statut des langues proclamait que I'anglais et le français sont les langues officielles pour tout ce qui relève du Parlement et du gouvernement du Canada (Loi sur les Langues officielles, abrogée en 1988). Mais au lieu de reconnaître les deux " peuples fondateurs ", le Premier ministre Pierre ElliotTrudeau créa la politique du multiculturalisme, assortie d'un désaveu de toute question nationale interne. II déclara devant le Parlement en 1971 : "Chaque groupe ethnique a le droit de préserver et de favoriser sa culture et ses valeurs propres dans le contexte canadien. Dire que nous avons deux langues officielles ne signifie pas que nous avons deux cultures officielles et aucune culture n'est plus officielle qu'une autre ". Comme le résume bien Tariq Modood : "Là où le multiculturalisme a été accepté et a fonctionné comme projet d'État ou projet national (au Canada, en Australie et en Malaisie, par exemple) il n'était pas une dimension accidentelle, mais faisait partie intégrante $d^{\prime}$ 'un projet de construction nationale " (Modood, $2007:$ 47).

La Constitution du Canada a été rapatriée de Londres en 1982, sans I'assentiment du Québec, et le multiculturalisme a été enchâssé dans la Charte canadienne des droits et libertés. La Loi sur le multiculturalisme canadien de 1988 consiste à " reconnaître le fait que le multiculturalisme est une caractéristique fondamentale de l'identité et du patrimoine canadien et constitue une ressource inestimable pour l'avenir du pays [...] parallèlement à l'affirmation du statut des langues officielles et à l'élargissement de leur usage, à maintenir et à valoriser celui des autres langues "(Canada, Gouvernement du Canada, 1988 : paragraphe. 3.1). Elle donne au gouvernement fédéral la possibilité d'invalider les lois des provinces (par exemple, la Charte de la langue française du Québec), au nom de la défense des droits des individus. Elle ouvre également la porte aux demandes de reconnaissance de la différence, exerçant du même coup des pressions auxquelles ni le système politique ni les élites ne peuvent donner suite (Rocher et Salée, 1993).

Dans un premier temps, le Canada s'est attaché à la préservation des folklores nationaux d'origine. Or, compte-tenu des critiques émanant de divers secteurs de la société civile, le gouvernement fédéral s'est recentré sur l'intégration sociale et économique, le redressement des discriminations, la réforme des institutions et les mesures de promotion sociale au nom de l'égalité des chances (McRoberts, 1997 ; Patel, 2007). II vise en même temps la revalorisation de la citoyenneté canadienne et la promotion de l'identité canadienne (Canada, CIC, 2014). Le ministre de la Citoyenneté et de I'Immigration, Jason Kenny, déclarait, en 2009, que le multiculturalisme ne doit pas devenir une " religion 
rigide ". Le ministère du Patrimoine canadien ne finance plus "les festivals, les camps, les activités religieuses ou la célébration de fêtes nationales étrangères et d'autres événements culturels ", ni les "initiatives portant sur les langues ancestrales " ou encore les " marches ou défilés commémoratifs ". Les priorités du programme concernent désormais l'intégration économique, le mentorat et l'éducation civique des jeunes à risque, les activités de promotion de " la compréhension interculturelle et des valeurs canadiennes ", tout en abordant des questions telles que "l'exclusion sociale de certaines communautés culturelles et la radicalisation ". Le ministre mettait en garde contre la formation d'enclaves ethniques: "On doit davantage miser sur les moyens de rapprocher les communautés pour s'assurer de ne pas voir surgir le genre d'enclaves ethniques que l'on retrouve dans certaines zones d'Europe "(Buzetti, 2009 : A3).

Par ailleurs, le Canada est tenu de déposer un rapport périodique au Comité pour l'élimination de la discrimination raciale des Nations unies. En 2001, issue de la $3^{\mathrm{e}}$ conférence de I'ONU sur le racisme, la Déclaration de Durban exhortait les États à " nommer et reconnaître " le racisme, à prévenir et à atténuer les effets néfastes de la mondialisation sur les populations marginalisées et racisées. Elle invitait les États à se doter de plans d'action et à respecter les engagements pris dans divers cadres. Dès lors, en 2005, le ministère du Patrimoine canadien dévoilait un Plan d'action canadien contre le racisme (Canada, Patrimoine canadien, 2005).

La notion de super-diversité se conjugue désormais avec celle du multiculturalisme au sein de la fonction publique fédérale ${ }^{3}$. Cette notion descriptive est attrayante, elle signifie mixité et complexité, mais elle suscite également des questions sur le type de gouvernance qu'elle implique : "Lorsque la mobilité des personnes s'accroît à l'échelle mondiale et que les gens deviennent des itinérants transculturels, comment la société et ses institutions peuvent-elles obtenir la coopération des Canadiens ? " (Canada, Horizons de politiques Canada, $2011:$ 1).

De leur côté, des chercheurs canadiens s'appliquent aussi à repenser le multiculturalisme, avec des argumentaires semblant aux antipodes. Selon Kymlicka, les "futurs incertains du multiculturalisme " s'expliquent par trois facteurs susceptibles d'amoindrir les appuis de la population au multiculturalisme : I'immigration illégale qui accrédite l'idée selon laquelle le Canada est incapable de contrôler ses frontières; I'utilisation du multiculturalisme par certains groupes pour défendre des pratiques non libérales ; le fait que l'on abuse de l'État provi-

\footnotetext{
3 « Selon les projections de Statistique Canada, d'ici à 2031, entre $29 \%$ et 32 \% des Canadiens pourraient appartenir à un groupe de minorité visible, $14 \%$ auraient une religion autre que le christianisme et presque $30 \%$ auraient une langue maternelle distincte de l'anglais et du français. Cette diversité récente se superpose à la charte historique des peuples du Canada constitués des Autochtones, des Anglais et des Français. La diversité du Canada se concentre en majorité dans les grandes agglomérations urbaines. En 2006, Toronto et Vancouver avaient des pourcentages de population d'origine étrangère plus élevés par rapport aux grandes villes d'Australie et des États-Unis. En 2006, 45,7 \% de la population de Toronto, 39,6 \% de celle de Vancouver et $20,6 \%$ de celle de Montréal était née à l'étranger, comparativement à 31,7\% à Sydney (Australie) et à 36,5 \% à Miami, en Floride. D'ici 2031, on prévoit que les minorités visibles représenteront $60 \%$ des populations de Toronto et de Vancouver, et $31 \%$ de la population de Montréal» (Canada, http://www.horizons.gc.ca/fra)
} 
dence, lequel a été construit grâce aux sacrifices des générations précédentes (2005 : 83). Les White European immigrants, comme les Polonais et les Italiens, n'étaient pas perçus comme une menace contre la civilisation occidentale. $\mathrm{Ni}$ les immigrants d'Amérique latine ou de la Caraïbe qui sont majoritairement des chrétiens. Ni les Chinois, ni les Japonais : "In the West today, therefore, it is primarily Muslims who are seen as raising this risk" (Ibid.).

À l'opposé, dans un numéro spécial de Canadian Ethnic Studies, Augie Fleras soutient que le multiculturalisme de mosaïque est obsolète, alors que le monde est maintenant fait de connexions et de flux qui traversent de multiples univers (multiple universes -within universes- (multiverse), lesquels transcendent les frontières et confrontent I'autorité des États post-nationaux (Fleras, 2011 : 18). Ceci signifie des réalités fluides, des identités hybrides, et une multiplicité d'appartenances et d'allégeances, alors que le multiculturalisme de mosaïque s'appliquait à une société composée de "groupes ethniques " bien différenciés (Ibid. : 19). Le Canada se situe dans une ère postnationale et post-multiculturelle. II accommode déjà des ethnicités principales : "Canada's major Ethnicities " (namely, Aboriginal peoples, nation minorities (Quebec) and multicultural (immigrant) minorities). II est maintenant fait de fusions, de fissions et de fissures. II est plus que multiculturel. II est aussi " multiracial, multiclass, multigendered, multisexual, multilingual, multireligious, multigenerational, multihistorical, multicitizenships, and so on ". II n'est plus non plus un pays d'immigrants mais de transmigrants : "To the contrary, it is but one node in a global network of transmigrants whose consciousness and identities are incresingly reconfigured withthin a framework of transnational social networks patterns of belonging, attachment, and loyalty" (Ibid: 25-28) et il doit se diriger maintenant vers un modèle alternatif de gouvernance du vivre-ensemble apte à prendre en compte la diversité de la diversité. Ce genre de rhétorique excessive démontre de façon exemplaire le type de discours " post " qui tend à s'imposer dans les universités nord-américaines.

Enfin, un courant de pensée inspiré de la critical race theory dénonce le fait que le multiculturalisme de l'État canadien masque la persistance des pratiques racistes, issues du passé colonial, qu'il s'agisse du cas des Premières Nations et de la Loi sur les Indiens, ou encore des programmes croissants de travailleurs étrangers temporaires.

\section{Évolution des termes désignant la majorité et les minorités dans le contexte canadien}

Les chercheurs s'aventurent rarement à définir la nation ou l'identité canadienne. Commentant la fragmentation induite par la politique du multiculturalisme, Howard-Hasmann a déjà soutenu qu'il existe une "identité ethnique canadienne " (définie par le territoire, la religion chrétienne, la langue anglaise) vers laquelle doivent converger les " groupes ethniques ancestraux " (HowardHassman, 1999). À l'opposé de ce type d'analyse, nombre de vocables désignent les minorités.

Depuis le XIXe siècle, le lexique identitaire canadien a évolué de la " race " à l'ethnicité. Les termes " nègre " (negro), " mulâtre " (mulato), " métis " et " sauvage " (Indian) figurent dans le recensement dès 1851. Le mot " nègre " est encore présent en 1961. Dans les années 1960, on a commencé à user du terme 
" groupes ethniques ". En 2006, parmi les " origines ethniques " recensées, on classait aussi bien les Italiens, les Juifs, les Africains, les Noirs, que les Québécois. Ce type de catégorisation ethnicise notamment les Inuits ou les Micmacs en contradiction avec la reconnaissance de leur statut de Premières Nations, ou encore les Québécois, également reconnus comme formant une nation par la Chambre des Communes à Ottawa, en 2006. Ce lexique étatique, produit à la suite de consultations avec les chercheurs, colore en retour leurs travaux. Ainsi, Augie Fleras confond les "Ethnicités " et les nations, alors que la plupart des spécialistes anglophones écrivent le mot "Québécois " avec accents, ce qui révèle leur ethnicisation particulière au sein de la fédération canadienne.

Cependant, la " race " persiste dans le discours gouvernemental, scientifique et public. Outre les expressions "groupe racial ", " minorité raciale ", " communauté raciale ", " race noire ", " minorités visibles ", le mot " race " figure une trentaine de fois dans le Plan d'action canadien contre le racisme de 2005. Le terme " minorités visibles " fait son apparition au cours de la décennie 1980 dans le contexte de la mise sur pied des programmes fédéraux d'équité en emploi. II désigne les " personnes autres que les Autochtones qui ne sont pas de race blanche ou qui n'ont pas la peau blanche et qui se reconnaissent comme telles auprès de leur employeur ". Ou encore : "Les groupes définis comme minorités visibles par la loi sont les Noirs et/ou les Antillais, les Chinois, les Sud-Asiatiques, les Arabes et les Ouest-Asiatiques, les Asiatiques du Sud-Est, les Latino-Américains et les individus originaires des îles du Pacifique ". Ce vocable qui porte des relents de l'idéologie raciste et masque les appartenances nationales d'une partie du monde global s'est malheureusement étendu de la sphère administrative à la sphère politique et publique (Labelle, 2011 : 54).

Et pourtant, le Comité pour l'élimination de la discrimination raciale des Nations unies met en garde sur les conséquences de son utilisation : "Tout en notant l'observation de l'État partie selon laquelle l'expression " minorités visibles " n'est employée que dans la loi sur l'équité en matière d'emploi et ne sert pas aux fins de la définition de la discrimination raciale, le Comité constate que cette expression est d'usage courant dans les documents officiels de l'État partie, y compris dans le cadre du recensement. Le Comité craint que l'usage de l'expression " minorités visibles " ne soit pas conforme aux buts et objectifs de la Convention (art. 1). Le Comité recommande à l'État partie de poursuivre sa réflexion sur les incidences de l'emploi de l'expression " minorités visibles " pour désigner " des personnes autres que les autochtones, qui ne sont pas de race blanche ou qui n'ont pas la peau blanche " (ONU, CERD, 2007 : par. 1).

\section{De I'interculturalisme et de l'antiracisme au Québec}

D'entrée de jeu, il faut souligner que tous les gouvernements du Québec, quel que soit le parti politique au pouvoir, ont rejeté le multiculturalisme canadien, au nom de la protection du français, langue officielle du Québec, ainsi que des caractéristiques politiques et culturelles de la nation québécoise, laquelle 
représente environ $23,2 \%$ de la population canadienne en $2010^{4}$. Le ministère de I'Immigration, de la Diversité et de I'Inclusion affirme que : " Le Québec constitue une nation qui exerce sa liberté à titre de société d'accueil. Le Québec est déterminé à promouvoir la vitalité de son caractère distinct et francophone et il entend faire de l'immigration une composante essentielle de sa vie collective. II agit librement en matière d'aménagement de sa diversité ethnoculturelle selon ses propres aspirations tout en respectant le partage des compétences constitutionnelles du régime fédéral canadien " (Québec, MIDI, 2014 : 9).

\section{L'interculturalisme au Québec}

L'immigration est un domaine de compétence partagée entre le gouvernement fédéral et les provinces. Depuis une trentaine d'années, l'État québécois défend une politique publique d'interculturalisme axée sur le rapprochement interculturel entre la " majorité francophone " et les " communautés culturelles ". La perspective de l'assimilation à la française n'a jamais eu bonne presse au Québec, la nation québécoise n'étant pas en position pour exercer le pouvoir d'attraction d'un État souverain. Déjà, la Charte de la langue française qui établissait le français comme langue officielle du Québec a provoqué d'importants conflits sociaux dans les années 1970. Elle ne fait toujours pas I'unanimité. Plusieurs de ses articles ont été déclarés inconstitutionnels par la Cour suprême du Canada et elle continue à faire l'objet d'un Quebec bashing systémique au Canada anglais de la part de ceux qui y voient une atteinte " tribale " aux libertés individuelles.

L'État québécois vise l'intégration, la mobilisation de la diversité et la promotion de la langue française (Québec, MICC, 2008 ; 2014) mais il n'a jamais adopté une loi sur l'interculturalisme. Le constitutionnaliste, Louis-Philippe Lampron, estime d'ailleurs que I'article 27 de la Charte canadienne des droits et libertés a pour effet de " court-circuiter toutes possibilités d'interprétation interculturelle des droits et libertés fondamentaux protégés sur le territoire québécois " puisqu'il prévoit " que l'interprétation des droits fondamentaux doit 'concorder avec l'objectif de promouvoir le maintien et la valorisation du patrimoine multiculturel des Canadiens' "(Lampron, 2010 : A7). Cependant, I'interculturalisme a donné lieu à une énumération de caractéristiques qui ont varié selon les documents ministériels : dialogue interculturel ; rapprochement interculturel ; culture québécoise de tradition française comme foyer de convergence rapprochement entre la majorité francophone et les diverses communautés ; culture publique commune, etc. (Rocher et al., 2007 ; Rocher et Labelle, 2010).

En 2007, le Québec a connu la " crise des accommodements raisonnables " (Labelle, 2014). Une Commission gouvernementale de consultation sur les pratiques d'accommodement reliées aux différences culturelles fut mise sur pied pour répondre aux préoccupations exprimées dans la foulée de certains événements ayant retenu l'attention des médias : port du kirpan par les Sikhs orthodoxes en milieu scolaire ; retrait du sapin de Noël à l'hôtel de ville de Montréal ;

\footnotetext{
4 Selon I'Enquête nationale auprès des ménages de 2011, le Québec compte 7903001 habitants dont 12,6\% sont nés à l'étranger. Én comparaison, la proportion d'immigrants au Canada est de 20,6\% et de $12 \%$ aux États-Unis. On dénombre au Québec 150 langues et plus de 200 religions. Parmi les Québécois, 80 \% ont le français comme langue maternelle, $8 \%$, I'anglais, $12 \%$, des langues tierces.
} 
lieux de prière dans les universités ; refus d'être servis par une femme dans les services publics; port de signes religieux dans la fonction publique; demandes de congés religieux ; prières dans les conseils municipaux ; etc. La Commission avait pour mandat de formuler des recommandations " pour que ces pratiques soient conformes aux valeurs de la société québécoise en tant que société pluraliste, démocratique et égalitaire " (Québec, CCPARDC, 2007). Un sondage réalisé, en décembre 2006 et en janvier 2007, par Léger Marketing rapportait que $83 \%$ des Québécois et $74 \%$ des " communautés culturelles " "croient que les immigrants devraient respecter les lois et les règlements du Québec, même si cela va à l'encontre de certaines croyances religieuses ou pratiques culturelles ", soit un écart assez mince (Ibid. : 67), ce qui n'a pas empêché la Commission d'opposer la majorité francophone aux minorités. Mais au-delà des pratiques $d^{\prime}$ 'accommodement, les auteurs du rapport se sont attachés à discuter d'interculturalisme, de laïcité et d'identité québécoise.

La Commission définit I'interculturalisme comme un modèle socioculturel propre au Québec et en établit les cinq traits principaux. L'interculturalisme : institue le français comme langue commune des rapports interculturels; cultive une orientation pluraliste soucieuse de la protection des droits ; préserve la nécessaire tension créatrice entre, d'une part, la diversité et, d'autre part, la continuité du noyau francophone et le lien social ; met un accent particulier sur l'intégration et la participation; et préconise la pratique des interactions (Québec, CCPARDC, 2008 : 121). Le rapport insiste sur l'insécurité culturelle des francophones (désignés alternativement comme Québécois canadiens-français, Québécois d'origine canadienne-française, francophonie majoritaire, majorité francophone) et leur sensibilité de minoritaires dans I'espace canadien (Ibid: 119), de telle sorte que I'un des commissaires, Gérard Bouchard, fera valoir un "principe de préséance de la majorité francophone ", soit les valeurs " historisées " ou "fondatrices " de la société québécoise, comme guide de la perspective interculturaliste (Bouchard, 2011).

Ayant mis l'accent sur les accommodements à caractère religieux, la Commission a inévitablement relancé le débat sur la laïcité. Sous l'influence du philosophe Charles Taylor, un défenseur du multiculturalisme, elle recommande un modèle de "laïcité ouverte " qui " ne sacrifie pas la séparation de l'État et de l'Église et la neutralité de l'État envers les religions au profit de la liberté de religion des croyants "(Québec, CCPARDC, $2008: 20$ ), avec restriction du port des signes religieux ostensibles pour certains représentants de la fonction coercitive de l'État. Cette position a suscité une vive opposition chez les Québécois de diverses origines pour qui tous les représentants de l'État doivent adopter un devoir de réserve aussi bien en matière de conviction religieuse que politique. Ils ont été accusés de vouloir importer un modèle à la française, antidémocratique, ayant pour effet pervers de provoquer l'exclusion des femmes portant le hijab de la fonction publique (Labelle, Couture et Remiggi, 2012 ; Labelle, 2014). Sur ce terrain, on a pu noter une diversité de positions à l'intérieur de la "diversité ", comme sur la question des accommodements raisonnables.

À l'automne 2013, le gouvernement du Parti Québécois déposait à l'Assemblée nationale un projet de Charte affirmant les valeurs de laïcité et de neutralité religieuse de l'État ainsi que d'égalité entre les femmes et les hommes et encadrant les demandes d'accommodement. Entre septembre 2013 et janvier 
2014, I'appui au projet de Charte était de $68 \%$ chez les francophones (donc aussi bien issus de la majorité que de certaines minorités) et $21 \%$ chez les non-francophones (Léger Marketing). Le projet fut abandonné par le gouvernement du Parti Libéral, élu en avril 2014. Un autre sondage CROP effectué en février 2015 montre que $51 \%$ des "Québécois " sont favorables à l'idée d'une charte de la laïcité réaffirmant les valeurs communes, bien qu'un clivage entre francophones (57\%) et non-francophones (27\%) s'observe (Boivin, 2015).

Le rapport de la Commission gouvernementale de consultation sur les pratiques d'accommodement reliées aux différences culturelles a également fourni matière à alimenter le débat sur la nation et le nationalisme, la citoyenneté et le rapport majorité/minorités (Labelle, Antonius et Toussaint, 2013). Selon le sociologue Gilles Bourque, la conceptualisation de la nation québécoise que présente la Commission " réunit d'abord tous les citoyens du Québec dans la nation québécoise, et les redistribue par la suite dans une multiplicité de groupes ethniques nettement différenciés ". Dans ce contexte, la question nationale québécoise s'est vue dépolitisée de telle sorte que "les luttes politiques sans cesse reprises dans I'histoire du Québec à propos de la définition de la communauté nationale et du statut du territoire deviennent des relations chaotiques et inquiètes entre des groupes ethniques ". Le malaise identitaire a donc été ramené à sa seule composante socio-psychologique et a reposé sur des considérations liées à l'insécurité ressentie par le "groupe ethnique " majoritaire (Bourque, 2008 : A6). Dans la même veine, le sociologue Guy Rocher reproche à la Commission d'avoir présenté une majorité francophone tantôt comme une minorité inquiète au sein de la fédération canadienne, tantôt comme une majorité flageolante, incertaine d'elle-même, sur le territoire du Québec. Les commissaires auraient nui à leur projet interculturel, même s'ils appelaient de leur vœu " l'institution d'un rapport majorité/minorités conforme à l'idéal interculturaliste " (Rocher, 2008 : A6). Bref, il aurait fallu analyser comment la Loi sur les langues officielles et la politique du multiculturalisme agissent comme contrepoids à la Charte de la langue française et à la perspective de l'interculturalisme. Alors que le multiculturalisme est indissociable de la valorisation de la citoyenneté canadienne, l'interculturalisme cherche à renforcer le sentiment d'appartenance au Québec dans un contexte de nation minoritaire où la citoyenneté demeure un impensé (Rocher et Labelle, 2010 ; Labelle et Rocher, 2011 ; Labelle, 2013).

\section{Les Québécois: une nation plurielle en situation minoritaire}

Le statut de nation minoritaire du Québec au sein de la fédération canadienne ne peut manquer d'influer sur la représentation publique du rapport majorité/ minorités.

En 2006, les députés de la Chambre des Communes à Ottawa adoptaient une motion reconnaissant que les "Québécois et les Québécoises forment une nation au sein d'un Canada uni ", court-circuitant ainsi les prétentions du Québec à l'autonomie gouvernementale et à l'indépendance. Or, dans I'esprit des médias du Canada anglais, ainsi que des experts du fédéralisme canadien, le mot "Québécois " renvoie le plus souvent aux seuls descendants des colons français qui forment la majorité sur le territoire québécois. D'un autre côté, si tous les gouvernements québécois défendent une définition civique et politique 
de la nation, l'ambiguïté persiste quant à la signification du terme "Québécois" au sein de l'État et dans l'espace public.

Le lexique identitaire en témoigne. À I'initiative du ministre et poète Gérald Godin, le ministère de I'Immigration, créé en 1968, changera d'appellation pour devenir le ministère des Communautés culturelles et de I'Immigration en 1981. Gérald Godin tenait à faire une distinction entre les immigrants et les " communautés culturelles ". Ces dernières étaient pour lui des Québécois d'implantation ancienne. II les considérait comme un atout pour le Québec et estimait qu'il fallait les appuyer dans l'affirmation de leurs particularités culturelles. Le vocable " communautés culturelles " a pénétré profondément la société québécoise. Avec le temps, sondeurs, chercheurs, politiciens, journalistes ont littéralement institutionnalisé le binôme "Québécois/communautés culturelles ". Plus récemment, la " diversité " tend à supplanter le mot " communautés culturelles " ainsi qu'en témoigne le document Vers une nouvelle politique québécoise en matière d'immigration, de diversité et d'inclusion, soumis pour consultation par le MIDI et on note un effort pour définir l'interculturalisme comme une approche originale "s'appuyant sur les principes de la reconnaissance mutuelle, de l'équité et de l'engagement partagé ainsi que sur les pratiques citoyennes participatives valorisant les contacts et le dialogue interculturels. Ces principes et ces pratiques visent à concrétiser les convictions profondes de la société à l'égard de l'égalité et de la dignité humaine et son aspiration à solidifier les liens de solidarité entre I'ensemble des Québécoises et des Québécois " (Québec, MIDI, $2014: 1$ ).

La représentation du rapport majorité/minorités sous la forme de blocs monolithiques en opposition mérite d'être déconstruite. Les sondages peinent à démontrer les convergences sur les grands enjeux de société, faute de terminologie adéquate. Et pourtant les enquêtes qualitatives de terrain démontrent que les inquiétudes suscitées par "I'arbitrage entre des croyances et des convictions de plus en plus variées, ainsi que leur expression dans l'espace public " (Québec, MIDI, 2014 : 18) ne sont pas seulement le fait d'une majorité francophone se percevant comme minoritaire, mais sont également partagées par de larges fractions de citoyens issus de l'immigration.

Parallèlement à la catégorisation Québécois/communautés culturelles, le rapport majorité/minorités persiste à se traduire sous le langage de la " race ", en référence à l'énumération des motifs contre lesquels il est interdit de discriminer, motifs mentionnés dans la Charte canadienne des droits et libertés et la Charte québécoise des droits et libertés de la personne. En 2008, dans La diversité une valeur ajoutée. Politique gouvernementale pour favoriser la participation de tous à l'essor du Québec, le MICC présentait la "race " comme une fausse notion, ce qui n'allait pas sans difficulté compte-tenu des pressions de certains groupes d'intérêt qui militent en faveur de son maintien dans la lutte contre le racisme (Labelle, $2011: 85$ ). En effet, pour illustrer le rapport dominant/ dominé, les associations de défense des minorités dont j'ai étudié le discours opposent elles aussi des totalités indifférenciées : Blancs/minorités visibles ; groupe majoritaire/minorités ; Québécois pure laine/minorités visibles, etc. (Ibid. : 102). Ce faisant, quelles que soient leurs dénominations, les minorités ethnoculturelles et racisées sont pensées comme étant à l'abri de la culture raciste et sexiste du système monde (Wallerstein, 1990). 


\section{Conclusion}

En dépit de l'effet de mode des notions de diversité, de super-diversité et de diversalité qui renvoient à de multiples variables sociologiques, et dont il faut évaluer en principe le poids relatif, le rapport majorité/minorités est traité essentiellement sous un angle culturaliste, ethnicisant et racialisant dans la littérature parcourue. La perspective de la citoyenneté étant quasiment absente, sinon pour être adjectivée, la communauté politique est ici représentée comme un univers de "communautés " structurellement encouragées à se reproduire. Ainsi, il est difficile de tenir compte et de restituer la diversité des positions politiques et idéologiques des minorités, qu'il s'agisse des modèles d'intégration, de la laïcité, de l'égalité des hommes et des femmes ou de la nation. Plutôt que de démontrer des convergences entre majorités et minorités, les décideurs, les chercheurs et les médias sont trop souvent à l'affût de la polarisation de leurs rapports, posée en postulat. Comme je l'ai mentionné en introduction de ce texte, I'analyse précédente ne concerne pas la question des nations autochtones (sinon par le biais de la racisation) dont la condition postcoloniale et le projet politique exigent un cadre interprétatif ne relevant ni du multiculturalisme, ni de I'interculturalisme.

Les majorités et les minorités ethnoculturelles et racisées sont la plupart du temps traitées sous l'angle démographique, ce qui entraîne des interprétations souvent hâtives des rapports de pouvoir. Or, un regard sociologique exige que l'on tienne compte des modes d'incorporation dans les sociétés d'accueil et des liens qui les lient dans le système monde. De plus, les catégories d'analyse des chercheurs devraient être distinguées des catégories de pratique qui servent à la mobilisation des groupes sociaux, ce qui demande " a self-reflexive stance towards our categories "(Brubaker, 2013a : 6). Ainsi, la mise en avant de la religion comme cadre de référence risque de se faire " at the expense of alternative and possibly more relevant frames of reference "(Brubaker, 2013a : 5 et 6). Car les problèmes d'incorporation sont souvent d'ordre économique et fondés sur le statut d'entrée, avant de relever de l'ethnicité, de la racisation ou de la religion.

Comment procéder à l'évaluation d'une gouvernance étatique multiculturaliste, interculturaliste et/ou antiraciste de façon générale ? Si l'on s'arrête au cas canadien et québécois, le discours, les choix stratégiques, les mesures et les indicateurs qui figurent dans les textes gouvernementaux abondent d'argumentaires qui révèlent la confusion des genres. On associe de manière systémique la gestion de la diversité, la promotion du multiculturalisme et la lutte contre le racisme dans le Plan canadien d'action contre le racisme. La politique québécoise procède au même amalgame : sensibilisation au racisme, éducation interculturelle, éducation aux droits et à la citoyenneté, reconnaissance et promotion de la diversité, formation interculturelle et antiraciste, etc. Sur le site internet du ministère de I'Immigration, de la Diversité et de I'Inclusion, on peut lire que la lutte contre le racisme doit s'intéresser à la discrimination basée sur la couleur, I'origine ethnique ou nationale, I'appartenance culturelle ou religieuse et l'homophobie.

Or ni le multiculturalisme ni l'interculturalisme ne peuvent répondre adéquatement aux problématiques soulevées par le racisme et sont souvent inattentifs 
aux structures de domination et d'exploitation, ainsi qu'aux rapports de classe. Ces philosophies politiques doivent être clairement distinguées dans le discours et dans les pratiques de l'État. À propos des luttes pour la reconnaissance, Cécile Laborde écrit : " C'est moins la reconnaissance identitaire que la résistance à I'assignation identitaire qui est souvent en jeu ". II s'agit de "luttes contre la domination et l'assignation identitaire, plutôt que comme (sic) des luttes pour la reconnaissance de l'identité " (Le Goff et Anctil, 2009 : 122). En effet, comme de nombreuses enquêtes de terrain l'ont démontré, les revendications de minorités racisées particulièrement vulnérables, selon les contextes et les conjonctures (Diène, 2006), sont avant tout des revendications d'égalité et de justice sociale. Comme le fait remarquer le Barreau du Québec : « il s'agit là d'enjeux différents, qui, bien que pouvant se compléter dans le cadre des actions de l'État, doivent être traités distinctement afin de ne pas restreindre ou diluer les obligations de l'État en matière de droit à l'égalité et de lutte contre la discrimination " (Labelle, 2011 : 166).

Un autre constat est que le multiculturalisme et l'interculturalisme coexistent fort bien avec un discours utilitariste portant sur la rentabilité des réseaux transnationaux des immigrants et des minorités. Ici, I'État prend acte des flux d'idées, de revendications et de moyens que véhiculent ces réseaux. Au fédéral comme au provincial, l'immigration est vue comme une des composantes de la mondialisation qu'on ne sous-estime plus. Un document intitulé Élaboration de l'analyse de rentabilisation du multiculturalisme, destiné à la Direction de l'action directe et promotion et à la Direction générale du multiculturalisme et des droits de la personne du ministère du Patrimoine canadien, incite à " parfaire l'analyse de rentabilisation de l'intégration des préoccupations relatives au multiculturalisme dans les politiques et les services des ministères "(Burstein, $2004: 3$ ). Pour I'auteur, les transilient immigrants font partie d'une nouvelle "classe créative ", apte à mobiliser leurs réseaux internationaux en vue d'investissements et de bonnes pratiques commerciales. Le ministère du Patrimoine canadien reconnaissait que les liens que les diverses "communautés culturelles et raciales" " entretiennent avec presque tous les pays du monde sont synonymes de prospérité économique et ont contribué à susciter l'intérêt du gouvernement du Canada à l'égard du multiculturalisme " (Canada, Patrimoine canadien, 2005).

Le Québec tient un discours analogue et estime que l'immigration contribue " au développement durable du Québec et de ses régions, dans le respect des valeurs communes "(Québec, MICC, 2011). II s'agit maintenant de mobiliser la diversité, car l'immigration a un impact sur l'innovation : élargissement de l'expertise québécoise sur la scène internationale, accès aux marchés étrangers, source importante de capitaux et d'investissements, contribution au dynamisme entreprenarial québécois, apport de main-d'œuvre dans des secteurs de pointe et dans les services, etc. (Québec, MIDI, 2014).

Enfin, la complexité des enjeux politiques est manifeste. La littérature semble assez muette sur la dimension politique des changements introduits par la mobilité internationale des migrants. Le rapport majorités-minorités est très différent dès lors que les minorités s'appuient sur l'une ou l'autre majorité pour négocier leur degré de participation à diverses communautés politiques, qu'il s'agisse du lieu de résidence ou du lieu d'origine dont on continue à se revendiquer. En dépit de la promotion du multiculturalisme ou de l'intercultu- 
ralisme dans le cadre de l'État nation, les modes d'incorporation ne se posent plus dans les mêmes termes en raison des liens et des intérêts transnationaux des minorités diasporiques, par exemple. Là où des conflits opposent la société d'accueil et le pays d'origine, cela peut entraîner des difficultés majeures comme c'est le cas présentement pour plusieurs sociétés occidentales confrontées à la radicalisation islamiste.

Quant à l'ethnicisation de la majorité québécoise, là aussi, la dimension politique est à considérer. Tant que le projet d'autonomisation de la nation québécoise divise les composantes autochtone, anglophone et francophone d'une entité étatique québécoise minoritaire, subordonnée à la nation canadienne, le rapport majorité-minorités au Québec comme au Canada reste ambigu. Les minorités au Québec se rapportent à quelle majorité : la majorité francophone québécoise ou la majorité anglophone canadienne ? À moins de se définir en marge de la minorité anglophone québécoise ? Ou en marge de leur propre nation d'origine, conçue alors comme territoire de référence ? D'un point de vue politique, on ne peut pas postuler l'existence d'une majorité unique. C'était déjà inadéquat compte tenu du caractère fédéral du système politique canadien, cela devient de plus en plus problématique dans le cadre d'un système d'échanges mondialisé traversé par des conflits d'allégeance qui ont de plus en plus tendance à dépasser les frontières du cadre étatique traditionnel.

\section{Références bibliographiques}

Alexander Jeffrey C. (2013) Struggling over the Mode of Incorporation: Backlash against Multiculturalism in Europe, Ethnic and Racial Studies, 36 (4), pp. 531-556

Amelina Anna and Faist Thomas (2012) De-naturalizing the National in Research Methodologies : Key Concepts of Transnational Studies in Migration, Ethnic and Racial Studies, 35 (10), pp. 1707-1724.

American Sociological Association (2003) The Importance of Collecting Data and Doing Social Scientific Research on Race, Washington, American Sociological Association, $15 \mathrm{p}$.

Antonius Rachad (2012) La polarisation du discours sur l'islam en Occident : quelques réflexions, in Micheline Labelle, Jocelyne Couture et Frank W. Remiggi Eds., La communauté politique en question. Regards croisés sur l'immigration, la citoyenneté, la diversité et le pouvoir, Québec, Presses de l'université du Québec, pp. 145-169.

Benhabib Seyla (2002) The Claims of Culture: Equality and Diversity in the Global Era, Princeton, Princeton University Press, 280 p.

Bennett David (1998) Multicultural States. Rethinking Difference and Identity, Londres et New York, Routledge, 320 p.

Boccagni Paolo (2012) Revisiting the "Transnational" in Migration Studies: A Sociological Understanding, Revue Européenne des Migrations Internationales, 28 (1), pp. 33-50.

Boivin Simon (2015) Un sondage CROP confirme que les Québécois comptent sur une charte pour les protéger, [en ligne] consulté le 21/02/2015. URL :http://www. lapresse.ca/le- soleil/actualites/politique/201502/20/01-4846120 
Bourque Gilles (2008) Bouchard-Taylor : un Québec ethnique et inquiet, Le Devoir, 30 juillet, p.A6.

Bourque Gilles (2008) L'insécurité d'un groupe ethnique, Le Devoir, 31 juillet, p. A.6.

Bouchard Gérard (2011) Qu'est-ce que l'interculturalisme?, Revue de droit de McGill, 56 (2), pp. 395-433.

Boyd Monica (1993) Mesurer I'origine ethnique dans les années à venir : populations, politiques et recherches en sciences sociales, in Gustave Goldmann et Nampeo McKenny Éds., Les défis que pose la mesure de l'origine ethnique : science, politique et réalité, Rapport, Conférence canado-américaine sur la mesure de l'origine ethnique, Statistique Canada et Bureau of the Census, Washington, D.C., U.S. Government Printing Office, pp. 529-545.

Brubaker Rogers (2001) The Return of Assimilation? Changing Perspectives on Immigration and its Sequels in France, Germany, and the United States, Ethnic and Racial Studies, 24 (4), pp. 531-546.

Brubaker Rogers (2013a) Categories of Analysis and Categories of Practice: a Note on the Study of Muslims in European Countries of Immigration, Ethnic and Racial Studies, 36 (1), pp. 1-8.

Brubaker Rogers (2013b) Language, Religion and the Politics of Difference, Nations and Nationalism, 19 (1), pp. 1-20.

Burstein Meyer (2004) Élaboration de l'analyse de rentabilisation du multiculturalisme, Direction de l'action directe et promotion, Direction générale du multiculturalisme et des droits de la personne, Ottawa, ministère du Patrimoine canadien, $39 \mathrm{p}$.

Buzetti Hélène (2009) Multiculturalisme : Ottawa veut célébrer l'intégration plutôt que la spécificité, Le Devoir, 11 février, p. A3.

Calhoun Greg et Wieviorka Michel (2013) Manifeste pour les sciences sociales, Penser Globa, 1, pp. 5-39.

Canada. Citoyenneté et Immigration Canada (2012) Découvrir le Canada. Les droits et responsabilités liées à la citoyenneté, Ottawa, Sa Majesté la reine du chef du Canada, représentée par le ministre de la Citoyenneté et Immigration Canada, $68 \mathrm{p}$.

Canada. Citizenship and Immigration Canada (2012) Promoting Integration, Annual Report on the Operation of the Canadian Multiculturalism Act, Ottawa, $42 \mathrm{p}$.

Canada. Citoyenneté et Immigration Canada (2014) Bâtir une société intégrée et cohésive sur le plan social. 2012-2013 Rapport annuel sur l'application de la loi sur le multiculturalisme canadien, Ottawa, Sa Majesté la reine du chef du Canada, représentée par le ministre de la Citoyenneté et Immigration Canada, $42 \mathrm{p}$.

Canada. Horizons de politiques Canada (2011) La superdiversité au Canada, 2 p. [en ligne] consulté le 17/06/2014. URL: http://www.horizons.gc.ca

Canada. Gouvernement du Canada (1988) Loi sur le multiculturalisme canadien, LRC (1985), ch. 24 (4 ${ }^{\mathrm{e}}$ suppl.). 
Canada. Patrimoine canadien (2005) Plan d'action canadien contre le racisme. Un Canada pour tous, Ottawa, ministre des Travaux publics et Services gouvernementaux Canada, $60 \mathrm{p}$.

Conseil de l'Europe (2008) Le livre blanc sur le dialogue interculturel. Vivre ensemble dans l'égale dignité, Strasbourg, Conseil de l'Europe, 70 p.

Conseil de l'Europe (2014) Identités et diversité au sein de sociétés interculturelles, Strasbourg, Conseil de l'Europe, 22 p.

Diène Doudou (2006) Le Racisme, la discrimination raciale, la xénophobie et toutes les formes de discrimination, Nations unies, Conseil économique et social, Commission des droits de I'homme, E/CN.4/2006/16, 22 p.

Faist Thomas (2012) Toward a Transnational Methodology: Methods to Address Methodological Nationalism, Essentialism, and Positionality, Revue européenne des migrations internationales, 28 (1), pp. 51-70.

Fistetti Francesco (2008) Théories du multiculturalisme, Paris, La Découverte, $227 \mathrm{p}$.

Fleras Augie (2012) From Mosaic to Multiversality: Repriming Multicultural Governance in a Post national Canada, Canadian Ethnic Studies, 48 (1-2), pp. 17-39.

Garner Steve (2007) Le paradigme de la whiteness et les identités blanches dans I'Angleterre contemporaine, in Michel Prum Éd., La fabrique de la race. Regard sur l'ethnicité dans I'aire anglophone, Paris, L'Harmattan, pp. 57-79.

Garner Steve et Fassin Eric (2013) 'Race', Sexualities and the French Public Intellectual: an Interview with Eric Fassin, Ethnic and Racial Studies, 36 (9), pp. 1465-1484.

Glick Schiller Nina (2010) A Global Perspective on Transnational Migration: Theorizing Migrațion without Methodological Nationalism, in Rainer Bauböck et Thomas Faist Éds., Diaspora and Transnationalism: Concepts, Theories and Methods, Amsterdam, Amsterdam University Press, p. 109-129.

Glick Schiller Nina and Salazar Noël B. (2013) Regimes of Mobility across the Globe, Journal of Ethnic and Migration Studies, 39 (2), pp.183-200.

Grosfoguel Ramón (2005) Les implications des altérités épistémiques dans la redéfinition du capitalisme global, Multitudes, 26, pp. 52-74.

Howard-Hassman Rhoda (1999) Canadian as an Ethnic Category: Implications for Multiculturalism and National Unity, Canadian Public Policy, 25 (4), pp. 523-537.

Isin Engin F. (2012) Citizenship after Orientalism: an Unfinished Project, Citizenship Studies, 16 (5-6), pp. 563-572.

Joppke Christian (2001) The Retreat from Multiculturalism in the Liberal State: Theory and policy, British journal of Sociology, 55, pp. 237-257.

Kymlicka Will (2005) The Uncertain Futures of Multiculturalism, Diversité canadienne, 4 (1), pp. 82-85.

Kymlicka Will (2010) The Rise and Fall of Multiculturalism? New Debates on Inclusion and Accommodation in Diverse Societies, International Social Science Journal, 61 (199), pp. 97-112. 
Kymlicka Will (2012) Comment on Meer and Modood, Journal of Intercultural Studies, 33 (2), pp. 211-216.

Labelle Micheline (1978; 1987) Idéologie de couleur et classes sociales en Haïti, Montréal, Presses de l'Université de Montréal, 393 p.

Labelle Micheline (2006) Un lexique du racisme : Étude sur les définitions opérationnelles du racisme et des phénomènes connexes, UNESCO, Coalition internationale des villes contre le racisme, Documents de discussion no. 1., Université du Québec à Montréal, Département de sociologie, Centre de recherche sur I'immigration, I'ethnicité et la citoyenneté, Les Cahiers de la CRIEC, 49 p.

Labelle Micheline (2008) Les intellectuels québécois face au multiculturalisme: hétérogénéité des approches et des projets politiques, Canadian Ethnic Studies, 40 (1-2), pp. 33-56.

Labelle Micheline (2011) Racisme et antiracisme au Québec. Discours et déclinaisons, Québec, Presses de l'université du Québec, 198 p.

Labelle Micheline, Antonius Rachad et Toussaint Pierre Éds. (2013), Les nationalismes québécois face à la diversité ethnoculturelle, Montréal, Éditions de I'Institut d'études internationales de Montréal, 319 p.

Labelle Micheline (2013) Quelle nation au-delà du principe de préséance de la majorité, in Micheline Labelle, Rachad Antonius et PierreToussaint Éds., Les nationalismes québécois face à la diversité ethnoculturelle, Montréal, Éditions IEIM, pp. 229-242.

Labelle Micheline et Rocher François (2011) Les limites indépassables de I'interculturalisme en contexte canadien : un chemin semé d'embûches, in Gérard Bouchard et al. Éds., L'interculturalisme. Dialogue Québec-Europe, Montréal, Actes du symposium international sur l'interculturalisme, 16 p., [en ligne], consulté le 23/02/2015. URL :www.symposium-interculturalisme.com/pdf/actes/ Chap8_1Labelle\%20et\%20Rocher.pdf>.

Labelle Micheline, Couture Jocelyne et Remiggi Frank W. Éds. (2012) La communauté politique en question. Regards croisés sur l'immigration, la citoyenneté, la diversité et le pouvoir, Québec, Presses de l'université du Québec, 375 p.

Lampron Louis-Philippe (2010) La gestion du pluralisme religieux au Québec. Comment déroger à la Charte canadienne sans déroger à la liberté de religion, Le Devoir, 8 mars, p. A7.

Le Goff Alice et Anctil Dave (2009) Entretien avec Cécile Laborde, Une revue multidisciplinaire sur les enjeux normatifs des politiques publiques et des pratiques sociales, 4 (2), pp. 112-129.

Lentin Alana and Titley Gavan (2014) The Crisis of Multiculturalism. Racism in a Neolibelral Age, London-New York, Zed Books.

Martiniello Marco et Adam Ilke (2013). Divergences et convergences des politiques d'intégration dans la Belgique multinationale. Le cas des parcours d'intégration pour les immigrés, Revue Européenne des Migrations Internationales, $29(2)$, pp. 77-93.

Martiniello Marco et Simon Pierre (2005) La catégorisation et la classification comme enjeux de pouvoir. Rapports de domination et luttes autour de la représentation dans les sociétés post-migratoires, Revue Européenne des Migrations Internationales, 21 (2), pp. 7-18. 
McRoberts Kenneth (1997) Misconceiving Canada. The Struggle for National Unity, Toronto, Oxford University Press, 288 p.

Meer Nasar and Modood Tariq (2012) How does Interculturalism Contrast with Multiculturalism?, Journal of Intercultural Studies, 33 (2), pp. 175-196.

Modood Tariq (2007) Rebâtir le multiculturalisme en Grande-Bretagne après les attentats du 7 juillet 2005, Éthique publique, 9 (1), pp. 40-49.

Neveu Catherine et al. (2012) Introduction: Questioning Citizenship. Questions de citoyennetés, Citizenship Studies, 5 (8), pp. 945-964.

Organisation des Nations unies. Comité pour l'élimination de la discrimination raciale (CERD) (2007) Examen des rapports présentés par les États parties conformément à I'article 9 de la Convention, Observations finales du comité, Canada, 25 mai (CERD/C/CAN/CO/18).

Parekh Bhikhu (2006) Rethinking Multiculturalism. Cultural Diversity and Political Theory, Basingstoke, Palgrave Macmillan, $400 \mathrm{p}$.

Patel Dhiru (2007) Myths, Realities and Challenges, in Sean P. Hier et B. Singh Bolaria Éds., Race and Racism in 21st Century Canada, Toronto, Broadview Press, pp. 257-274.

Québec. Commission de consultation sur les pratiques d'accommodement reliées aux différences culturelles (2007) Accommodements et différences. Vers un terrain d'entente, Québec, Commission de consultation sur les pratiques d'accommodement reliées aux différences culturelles, 44 p.

Québec. Commission de consultation sur les pratiques d'accommodement reliées aux différences culturelles (2008) Fonder l'avenir. Le temps de la conciliation, Québec, Commission de consultation sur les pratiques d'accommodement reliées aux différences culturelles, 22 mai, 310 p.

Québec. Ministère de I'Immigration, de la Diversité et de I'Inclusion (2014) Vers une nouvelle politique québécoise en matière d'immigration, de diversité et d'inclusion. Cahier de consultation, Québec, Gouvernement du Québec, Consultation publique 2015, $70 \mathrm{p}$.

Québec. Ministère de I'Immigration et des Communautés culturelles (2008) La diversité : une valeur ajoutée. Politique gouvernementale pour favoriser la participation de tous à l'essor du Québec, Montréal, Direction des affaires publiques et des communications, juin, $51 \mathrm{p}$.

Québec. Ministère de I'Immigration et des Communautés culturelles (2011) L'apport de l'immigration au développement durable, Consultation 20112015, Québec, Gouvernement du Québec, 9 p.

Rocher François et al. (2007) Le concept d'interculturalisme en contexte québécois: généalogie d'un néologisme, rapport présenté à la Commission de consultation sur les pratiques d'accommodement reliées aux différences culturelles, Montréal, Chaire de recherche en immigration, ethnicité et citoyenneté, Université du Québec à Montréal, 21 décembre, 63 p.

Rocher François et Labelle Micheline (2010) L'interculturalisme comme modèle d'aménagement de la diversité: Compréhension et incompréhension dans I'espace public québécois, in Bernard Gagnon Éd, La diversité québécoise en débat: Bouchard, Taylor et les autres, Montréal, Québec-Amérique, pp. 179-203. 
Rocher François et Salée Daniel Salée (1993) Démocratie et réforme constitutionnelle : discours et pratique, Revue internationale d'études canadiennes, 7-8, pp.167-185.

Rocher Guy (2008) Rapport Bouchard-Taylor : une majorité trop minoritaire?, Le Devoir, 12 juin, p. A.6.

Salée Daniel (2007) The Quebec State and the Management of Ethnocultural Diversity: Perspectives on Ambiguous Record, dans Keith Banting, Tom J. Courchesne et Leslie Seidle (dir.), Belonging? Diversity, Recognition, and Shared Citizenship in Canada, Montréal, Institut de recherche en politique publique, pp. 105-142.

Shachar Ayelet (2014) Introduction: Citizenship and the 'Right to Have Rights', Citizenship Studies, 18 (2), pp. 114-124.

Thobani Sunera (2007) Exalted Subjects. Studies of the Making of Race and Nation in Canada, Toronto, Toronto University Press, p. 391

Vertovec Steven (2007) Super-diversity and its Implications, Ethnic and Racial Studies, 30 (6), pp. 1024-1054.

Wallerstein Immanuel (1990) Culture as the Ideological Battleground of the Modern World-System», Theory, Culture \& Society, 7 (2), pp. 31-55.

Wallis Maria and Fleras Augie (2009) The Politics of Race in Canada, Don Mills, Oxford University Press, 312 p.

Wieviorka Michel (1998) Is Multiculturalism the Solution?, Ethnic and Racial Studies, 21 (5), pp. 881-910.

Wieviorka Michel (2011) Pour la prochaine gauche, Paris, Robert Laffont, 300 p.

Wieviorka Michel (2012) Multiculturalism: a Concept to be Redefined and certainly not Replaced by the extremely Vague Term of Interculturalism, Journal of Intercultural Studies, 33 (2), pp. 225-231. 


\section{Micheline Labelle}

\section{Multiculturalisme, interculturalisme, antiracisme : le traitement de l'altérité}

L'article examine les lignes de tension entre les analyses paradigmatiques du multiculturalisme, de l'interculturalisme et de l'antiracisme, en insistant sur le traitement du rapport majorité/minorités. L'auteure montre, dans une première partie, que ces philosophies politiques ne font pas seulement débat entre elles mais qu'elles sont jugées dépassées et inopérantes par les théories "post " de tous genres qui se déploient aux marges du champ traditionnel de l'immigration et des " études ethniques ". Dans une deuxième partie, elle analyse ces questions dans le contexte du fédéralisme canadien. Une troisième partie porte sur le cas québécois où la gouvernance de l'interculturalisme et de l'antiracisme tente de s'imposer dans un contexte de nation minoritaire. L'auteure fait ainsi ressortir les enjeux analytiques et politiques qui se posent pour les chercheurs et la gouvernance étatique de la diversité.

\section{Multiculturalism, Interculturalism, Antiracism: Analysing Otherness}

This article examines the areas of tension between paradigmatic analyses of multiculturalism, interculturalism and antiracism, with a focus on majority/ minority relations. The author demonstrates that, not only do these political philosophies disagree amongst themselves, but they are considered outdated and ineffective by a range of "post" theories which spring up on the margins of the traditional field of immigration and "ethnic studies." In the second section, she analyses these questions in the context of Canadian federalism. The third part bears on the Québec case where attempts at the governance of interculturalism and antiracism occur in the context of a minority nation. She highlights the analytical and political issues of concern to researchers and those involved in state governance of diversity.

\section{Multiculturalismo, interculturalidad, antirracismo: el tratamiento de la alteridad}

El artículo explora las tensiones que se generan entre las líneas de análisis paradigmático del multiculturalismo, de la interculturalidad, y del antirracismo, insistiendo particularmente en el tratamiento de la relación mayoría/minorías. La autora demuestra, en primer lugar, que esas filosofías políticas no solamente generan debate entre ellas, sino que además son juzgadas como inoperantes por todos los tipos de teorías "post" que se despliegan en los márgenes del campo tradicional de la inmigración y de los "estudios étnicos". En una segunda parte, estas cuestiones son analizadas en el contexto del federalismo canadiense. La tercera parte examina el caso de Quebec donde el buen gobierno de la interculturalidad y del antirracismo intenta imponerse en un contexto de nación minoritaria. La autora enfatiza los desafíos analíticos y políticos que estas cuestiones representan para los investigadores y para la gobernabilidad de la diversidad. 